Origin of Cometary Light and Heat.-Emile Delaurier advances the hypothesis that the great rapidity of cometary motion causes the æthereal matter to vibrate and transforms it into light and heat. He expands his hypothesis so as to accomnt for all the variations of form which the great comets undergo, for the apparent solar repulsion and for the curvature of the tail.--Les Mondes.

C.

\title{
Application of Electricity to the Study of Rapid Phe-
} nomena. - Marcel Deprez has contrived a register which gives excellent results in making rapid measurements with the intervention of a single Buwsen cell. He has already surceeded in reducing the time of a double signal to about $\frac{1}{1600}$ of a second. It is not necessary to have the current closed longer than for $\frac{1}{0}$ of of a second in order to give the signals. - Lumirir Electrique.

C.

Comet Spectrum. - The French ohservers find many striking resemblances between the spectra of Gould's and Coggia's eomets. The nucleus gives a continuous spectrum, without bands or lines. The nebulosity near the nuclens shows three bands, one very bright, the others faint. The band spectrum is so much like that of alcohol that Thollon considers them identical. The violet alcohol band is not seen, but its absence seems to be owing to atmospheric absorption. There is little doubt of the presen'e of carbon in some of its compounds.-Comptes: Rendus.

(!.

Explosion of Bubbles.-Plate:tu has published some new experiments upon thin liquid films. One of the most interesting is one which furnishes conclusive evidence of the contraction which the bubble undergoes during its rapid destruction. He filled with tobacco smoke a bubble of glyceric liquid of about 11 centimetres $(4 \cdot 3 \mathrm{in}$.) in dianeter and laid it upon a ring of 4 centimetres; waiting until the blue color of the summit showed that it was upon the point of bursting he pierced the summit with a metallic wire. The mass of smoke was thrown vertically for about a decimetre above the bubble and then spread horizontally, like an umbrella, and continued to mount more slowly while diffusing itself into the air. The experiment was repeated several times with the rame result.-Acad. Roy. de Belge. 